\title{
On the Rotational Contributions to the Dipole-Moment Derivatives
}

\author{
A. J. van Straten and W. M. A. Smit \\ Analytical Chemistry Laboratory, State University of Utrecht, Croesestraat $77^{A}$, Utrecht, \\ The Netherlands
}

\begin{abstract}
In order to get dipole-moment derivatives, $\partial \mathbf{u} / \partial S_{j}$, that are free from rotational contributions, we used Crawford's method applied to a new type of reference molecule. The agreement with earlier calculated rotational correction terms is good, the applicability of the new reference molecule is wider. The rotational contributions to the $\partial \boldsymbol{u} / \partial S_{j}$-quantities are presented for a number of $C_{2 v^{-}}$and $C_{3 v^{-}}$-type molecules.
\end{abstract}

\section{INTRODUCTION}

It is useful to interpret the experimental infrared absorption intensities in terms of dipole-moment derivatives with respect to the symmetry coordinates.

In the case of a molecule with a nonvanishing permanent moment, the motions represented by those symmetry species that include a molecular rotation produce $\partial \mathbf{u} / \partial S_{j-}$ quantities containing a contribution from the rotation of the permanent dipole moment.

In comparing data on different isotopic species or on closely related compounds, the only appropriate intensity parameters are those which are corrected for rotational contributions.

This contribution arises from the condition that the angular and linear momentum of the entire molecule is conserved in a perturbation of the equilibrium configuration under vibration. This requires any alteration in the angular or linear momentum, resulting from a symmetry distortion, to be exactly compensated by an amount of rotation or translation of the whole molecule with the same frequency. Since translational motions do not affect the dipolc moment of a molecule, only the rotational motions need be considered. As we know from pure rotational spectra, a molecular rotation contributes to the absorption intensity if the molecule has a permanent moment.

In the derivation of the intensity sum rules Crawford (1) has given the mathematical framework to calculate the difference in rotational contribution between two isotopic molecules. Subsequently Dickson, Mills and Crawford (2) proposed a method to obtain the absolute rotational correction for $\partial u / \partial S$-quantities. However, the choice of the rotational free reference molecule needed for such a calculation leads to difficulties in some cases.

Here we present a new type of reference molecule, with a wider applicability.

\section{THE ROTATIONAL CORRECTION}

The experimental integrated intensity, $A_{i}$, of the $i$ th fundamental absorption band is related to the square of the dipole derivative, $\partial u / \partial Q_{i}$, with respect to the $i$ th normal 
coordinate, $Q_{i}$, by

$$
A_{i}=\left(N_{0} \pi g_{i} / 3 c^{2}\right)\left(\partial u / \partial Q_{i}\right)^{2}
$$

in the harmonic-oscillator-linear-dipole approximation (3). In Eq. (1) $N_{0}$ is Avogadro's number, $c$ the speed of light, $\boldsymbol{u}$ the molecular dipole-moment vector, and $g_{i}$ the degree of degeneracy of the $i$ th fundamental.

The normal coordinates are defined in terms of a set of internal coordinates, $\mathbf{R}$, through the L-matrix: $\mathbf{R}=\mathbf{L Q}$ (4). Thus we can write

or in matrix notation,

$$
\frac{\partial \mathbf{u}}{\partial Q_{i}}=\sum_{j} L_{j i} \frac{\partial u}{\partial R_{j}},
$$

$$
(\partial \mathbf{u} / \mathbf{\partial Q})=\tilde{\mathbf{L}}(\partial \mathbf{u} / \mathbf{\partial R})
$$

$\tilde{L}$ being the transposed $L$-matrix.

In the interpretation of intensity data one usually determines the dipole-moment derivatives with respect to symmetry coordinates (5), which are taken as linear combinations of the internal coordinates, $\mathbf{S}=\mathbf{U R}$, leading to

$$
(\partial \mathbf{u} / \partial \mathbf{Q})=\tilde{\mathbf{L}} \tilde{U}(\partial \mathbf{u} / \partial \mathbf{S}) \text {. }
$$

Our aim is to separate the contribution originating from the compensating rotation accompanying a vibration from the dipole-moment derivatives, obtained from Eq. (2) or (3). This requires the availability of a reference molecule in which vibration produces no compensating rotatory motion. The definition of such a reference molecule is given in the following section.

The dipole-moment derivatives obtained from Eq. (3) will be denoted by ( $\mathbf{\partial u} / \mathbf{\partial S})_{A}$, where the subscript $A$ refers to the actual molecule under consideration. The theoretical quantity needed will be denoted by $(\boldsymbol{\partial} \boldsymbol{u} / \partial \mathbf{S})_{R}$, where the subscript $R$ stands for the reference molecule. The difference between these two parameters is the rotational correction, or in formula:

$$
(\partial \mathfrak{u} / \partial \mathbf{S})_{R}=(\partial \mathfrak{u} / \partial \mathbf{S})_{A}-\mathbf{V}_{A R} \text {. }
$$

The columnvector, $V_{A R}$, can be evaluated with the help of two additional coordinate sets :

$\mathbf{X}$ : the vector of the $3 N$ Cartesian displacement coordinates ( $N$ is the number of atoms in the molecule).

$\mathrm{g}$ : the external coordinates (the six translations and rotations). The transformation matrices between $\mathbf{g}, \mathbf{R}, \mathbf{S}$, and $\mathbf{X}$ are defined as follows.

$$
\begin{aligned}
& \mathbf{R}=\mathbf{B X}, \quad \mathbf{B}=(\mathrm{a} \mathbf{R} / \mathbf{\partial X}), \quad \mathbf{A}^{*}=(\mathbf{\partial X} / \mathbf{\partial R})^{*}, \\
& \mathbf{S}=\text { UBX }, \quad \text { UB }=(\partial \mathrm{S} / \partial \mathrm{X}), \quad \mathbf{A}^{*} \widetilde{\mathrm{U}}=(\partial \mathrm{X} / \partial \mathrm{S})^{*}, \\
& \boldsymbol{\varrho}^{*}=\underline{\beta}^{*} \mathbf{X}, \quad \underline{\beta}^{*}=\left(\partial \underline{\varrho}^{*} / \partial \mathrm{X}\right), \quad \boldsymbol{\alpha}^{*}=\left(\partial \mathrm{X} / \partial \mathrm{g}^{*}\right) \text {. }
\end{aligned}
$$

The matrices and vectors marked with an asterisk are isotope-dependent. The properties of these transformation matrices for a given isotope are:

$$
\begin{aligned}
& \mathbf{B} \boldsymbol{\alpha}^{*}=\mathbf{0}, \quad \mathbf{B}^{*} \mathbf{A}^{*}=\mathbf{0}, \quad \mathbf{B M}^{*-1} \tilde{\mathbf{B}}=\mathrm{G}^{*}, \\
& \mathbf{B A}^{*}=\mathrm{E}_{3 N-6}, \quad \boldsymbol{\beta}^{*} \boldsymbol{\alpha}^{*}=\mathbf{E}_{6}, \quad \boldsymbol{\beta}^{*} \mathbf{M}^{*-1} \tilde{\boldsymbol{\beta}}^{*}=\mathbf{N}^{*}, \\
& \mathbf{A}^{*} \mathbf{B}+\boldsymbol{\alpha}^{*} \boldsymbol{3}^{*}=\mathbf{E}_{3 N}, \quad \tilde{\boldsymbol{\alpha}}^{*} \mathbf{M}^{*} \boldsymbol{\alpha}^{*}=\mathbf{N}^{*-1},
\end{aligned}
$$


where $\mathbf{G}$ is Wilson's kinetic-energy matrix ( 4 ), $\mathbf{M}$ is the diagonal matrix of the atomic masses, and $\mathbf{N}$ is a $6 \times 6$ diagonal matrix containing the reciprocals of both the molecular mass and the principal moments of inertia.

Crawford (1) derived a relation between the A-matrices of two different isotopes. For the $\mathbf{A}$-matrix of the reference molecule this relation reads

$$
\mathbf{A}_{R}=\mathbf{A}_{A}-\alpha_{R} \boldsymbol{\beta}_{R} \mathbf{A}_{A},
$$

where the subscripts have the meaning as defined above.

From Eq. (7) one easily obtains

$$
\mathbf{U} \tilde{\mathbf{A}}_{R}(\partial \mathbf{u} \mathbf{u} / \mathbf{\partial X})=\mathbf{U} \tilde{\mathbf{A}}_{A}(\mathbf{\partial u} / \mathbf{\partial X})-\mathbf{U} \tilde{\mathbf{A}}_{A} \tilde{\boldsymbol{\beta}}_{R} \tilde{\boldsymbol{\alpha}}_{R}(\mathbf{\partial u} \mathbf{u} / \mathbf{\partial X}),
$$

which is equivalent to Eq. (4). So the column vector of the rotational corrections can be written as

$$
\mathbf{V}_{A R}=\left\{\mathrm{U} \tilde{\mathbf{A}}_{A} \tilde{\boldsymbol{\beta}}_{R}\right\}\left\{\tilde{\boldsymbol{\alpha}}_{R}(\partial \mathbf{\partial u} / \partial \mathbf{X})\right\} .
$$

Since translational motions do not change $\boldsymbol{u}$, we may rewrite the second part of $\mathbf{V}_{A R}(1)$, giving

$$
\tilde{\boldsymbol{\alpha}}_{R} \frac{\partial \boldsymbol{u}}{\partial \mathbf{X}}=\left(\begin{array}{c}
0 \\
0 \\
0 \\
\left(\mu_{0}\right)_{y} \mathbf{k}-\left(\mu_{0}\right)_{z} \mathbf{j} \\
\left(\mu_{0}\right)_{z} \mathbf{i}-\left(\mu_{0}\right)_{x} \mathbf{k} \\
\left(\mu_{0}\right)_{x} \mathbf{j}-\left(\mu_{0}\right)_{y} \mathbf{i}
\end{array}\right),
$$

where $\left(\mu_{0}\right)_{x}$ denotes the $x$-component of the permanent dipole moment and $\mathbf{i}, \mathbf{j}$, and $\mathbf{k}$ are the Cartesian unit direction vectors. In the case of symmetry this column vector

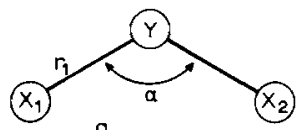

a.

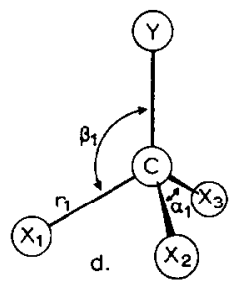

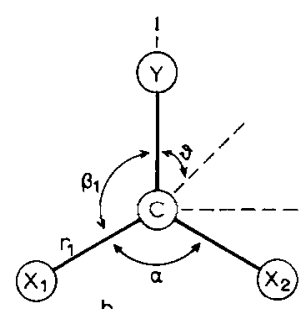

b.

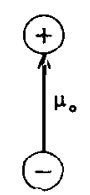

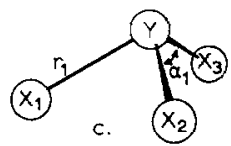

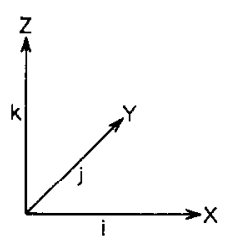

FIG. 1. Definition of internal coordinates in (a) bent $X_{2} Y$ molecules, (b) $X_{2} C Y$ molecules, (c) pyramidal $X_{3} Y$ molecules, (d) $X_{3} C Y$ molecules. Definition of dipole-moment direction and Cartesian coordinate axes. 
Table I. Rotational corrections and symmetry coordinates in methyl ha1ides. ${ }^{\text {a }}$

\begin{tabular}{|c|c|c|c|c|c|c|c|}
\hline \multicolumn{8}{|c|}{ 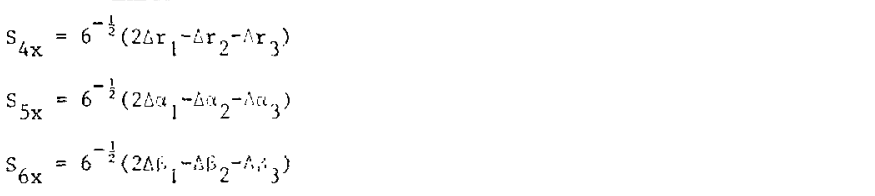 } \\
\hline \multirow[t]{2}{*}{$\mathrm{x}_{3} \mathrm{CY}$} & \multirow[t]{2}{*}{$\mu_{0}(D)$} & \multicolumn{3}{|c|}{$\begin{array}{l}\text { Reference molecule } \\
\text { with } m_{X}=0\end{array}$} & \multicolumn{3}{|c|}{$\begin{array}{l}\text { Reference molecule } \\
\text { with } \mathrm{m}_{\mathrm{C}}, \mathrm{m}_{\mathrm{Y}} \times 1000\end{array}$} \\
\hline & & $v_{4 x}$ & $v_{5 x}$ & $v_{6 x}$ & $v_{4 x}$ & $v_{5 x}$ & $v_{6 x}$ \\
\hline $\mathrm{H}_{3} \mathrm{CF}$ & -1.81 & +0.0798 & +0.0894 & -0.2085 & +0.0797 & +0.0892 & -0.3082 \\
\hline $\mathrm{D}_{3} \mathrm{CF}$ & -1.81 & +0.1123 & +0.1312 & -0.3220 & +0.1120 & +0.1310 & -0.3214 \\
\hline $\mathrm{H}_{3} \mathrm{CCl}$ & -1.87 & +0.0711 & +0.0685 & -0.1310 & +0.0710 & +0.0684 & -0.1308 \\
\hline $\mathrm{n}_{3} \mathrm{CCl}$ & -1.87 & +0.1073 & +0.1052 & -0.2073 & +0.1071 & +0.1050 & -0.2069 \\
\hline $\mathrm{H}_{3} \mathrm{CBr}$ & -1.80 & +0.0651 & +0.0586 & -0.1003 & +0.0650 & +0.0586 & -0.1002 \\
\hline $\mathrm{D}_{3} \mathrm{CBr}$ & -1.80 & +0.1009 & +0.0914 & -0.1587 & +0.1007 & +0.0913 & -0.1584 \\
\hline $\mathrm{H}_{3} \mathrm{CI}$ & -1.62 & +0.6542 & +0.0471 & -0.0755 & +0.0541 & +0.0471 & -0.0754 \\
\hline $\mathrm{D}_{3} \mathrm{CI}$ & -1.62 & +0.0850 & +0.0742 & -0.1199 & +0.0849 & +0.0740 & -0.1197 \\
\hline
\end{tabular}

often reduces to a very simple form, e.g., in the case of $C_{2 v}$ or $C_{3 v}$ symmetry there is only a nonzero component of the permanent moment along the main axis of symmetry.

The $\mathbf{U} \tilde{\mathbf{A}}_{A} \tilde{\mathbf{Z}}_{R}$ term gives the translation and rotation set up in the reference molecule, when its atoms execute the displacements appropriate to a symmetry distortion $S$ in the actual molecule. The $\mathbf{A}_{A}$-matrix can be calculated from (1)

$$
\mathbf{A}_{A}=\mathbf{M}_{A}{ }^{-1} \tilde{\mathbf{B}} \mathbf{G}_{A^{-1}} \text {. }
$$

The $\beta_{R}$-matrix can be written easily.

Thus, after defining an appropriate reference molecule the calculation of the rotational contributions is straightforward.

\section{THE REFERENCE MOLECULE}

The determination of the rotational corrections is only possible if we can define a reference molecule (a hypothetical molecule, being an isotope of the original one), which possesses rotation-free internal coordinates. 
Table II. Definition of symetry coordinates and rotational contributions in $\mathrm{Br}_{3} \mathrm{CH} / \mathrm{D}$.

\begin{tabular}{|c|c|c|c|c|c|c|}
\hline \multicolumn{7}{|c|}{$\begin{array}{l}s_{4 x}=6^{-\frac{1}{2}}\left(2 \Delta \beta_{1}-\Lambda \beta_{2}-\Delta \beta_{3}\right) \\
s_{5 x}=6^{-\frac{1}{2}}\left(2 \Delta r_{1}-\Delta r_{2}-\Delta r_{3}\right) \\
s_{6 x}=6^{-\frac{1}{2}}\left(2 \Delta \alpha_{1}-\Delta \alpha_{2}-\Delta \alpha_{3}\right)\end{array}$} \\
\hline \multirow[t]{2}{*}{$\mu_{0}(1)$} & \multicolumn{3}{|c|}{$\begin{array}{l}\text { Reference molecule } \\
\text { with } \mathrm{m}_{\mathrm{Br}}=0\end{array}$} & \multicolumn{3}{|c|}{$\begin{array}{l}\text { Reference molecule with } \\
\mathrm{m}_{\mathrm{C}}, \mathrm{m}_{\mathrm{H} / \mathrm{D}} \times 100000\end{array}$} \\
\hline & $v_{4 x}$ & $v_{5 x}$ & $v_{6 x}$ & $v_{4 x}$ & $v_{5 x}$ & $v_{6 x}$ \\
\hline $\mathrm{Br}_{3} \mathrm{CH}+0.99$ & +0.8049 & +0.1251 & -0.0104 & +0.8011 & +0.1251 & -0.0097 \\
\hline $\mathrm{Br}_{3} \mathrm{CD} . \quad+0.99$ & +0.8014 & +0.1229 & -0.0123 & +0.7994 & +0.1229 & -0.0119 \\
\hline
\end{tabular}

The only rotation-free molecule known in the literature is a molecule in which isotopes of zero mass are introduced for atoms which are not situated on the main molecular symmetry axis. For example, in the case of $X_{3} C Y$-molecules $(2,6)$, where $X, Y=\mathrm{H}, \mathrm{D}$, $\mathrm{F}, \mathrm{Cl}, \mathrm{Br}$, and $\mathrm{I}$, one has used as a reference molecule the isotopic molecule containing zero $X$-masses. During all vibrations of such a reference molecule the $C Y$-bond will maintain a fixed direction and thus there is no compensating rotational motion. Also

Table III. Bent triatomic molecules $x_{2} 7$.

\begin{tabular}{|lllll|l|}
\hline $\mathrm{S}_{3}=2^{-\frac{1}{2}}\left(\Delta \mathrm{r}_{1}-\Delta \mathrm{r}_{2}\right)$ \\
\hline $\mathrm{X}_{2} \mathrm{Y}$ & $\mathrm{r}_{\mathrm{XY}}(\mathrm{R})$ & $\alpha\left(^{0}\right)$ & Ref. & $\mu_{0}(D)$ & $v_{3}(\mathrm{D} / \mathrm{R})$ \\
& & & & & $\mathrm{m}_{3} \times 1000$ \\
\hline $\mathrm{H}_{2} \mathrm{O}$ & 0.9584 & 104.45 & 14 & -1.85 & -0.0771 \\
$\mathrm{D}_{2} \mathrm{O}$ & 0.9584 & 104.45 & 14 & -1.85 & 0.1436 \\
$\mathrm{H}_{2} \mathrm{~S}$ & 1.34 & 92.33 & 15 & -0.97 & -0.0156 \\
$\mathrm{D}_{2} \mathrm{~S}$ & 1.34 & 92.33 & 15 & -0.97 & -0.0302 \\
$\mathrm{O}_{2} \mathrm{~S}$ & 1.433 & 119.33 & 15 & +1.47 & +0.1810 \\
\hline
\end{tabular}


Table IV. Definition of symmetry coordinates and rotational contributions in $\mathrm{X}_{2} \mathrm{CY}$-molecules.

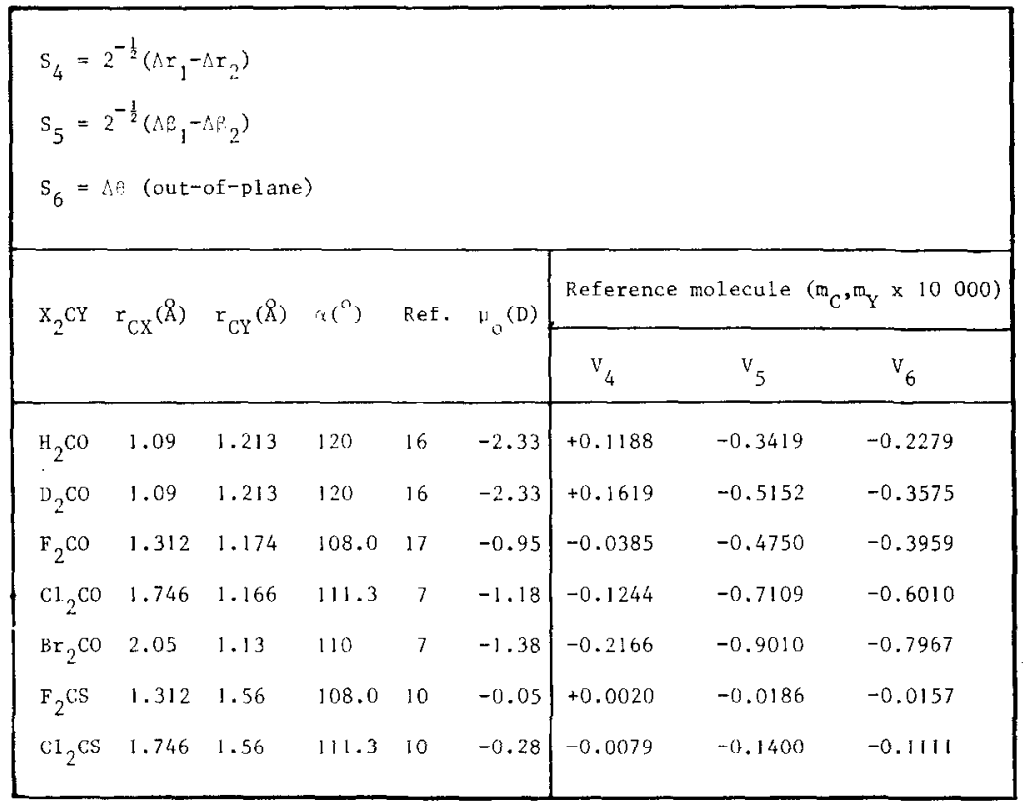

in the case of $X_{2} C Y(7-11)$ molecules $(X=\mathrm{H}, \mathrm{D}, \mathrm{F}, \mathrm{Cl}, \mathrm{Br} ; Y=\mathrm{O}, \mathrm{S})$ the introduction of an $X$-isotope of zero mass provides a usable reference molecule.

However, this procedure cannot be followed in bent $X_{2} Y$-or pyramidal $X_{3} Y$-molecules. Introduction of an $X$-isotope with zero mass in such molecules leads to indefinite elements of the $\boldsymbol{\beta}_{R}$-matrix, thus prohibiting the calculation of the rotational corrections (see Eq. (9)).

In order to overcome these difficulties, we propose the following reference molecule. Replace the atoms of the actual molecule which are situated on the main symmetry axis by isotopes that are a factor of a thousand or more heavier than the original atoms. ${ }^{1}$ In such a reference molecule, the compensating rotational motions are negligable, because of the large masses of the substituted isotopes. This is also valid in the case of $X_{2} Y$ - and $X_{3} Y$-molecules, while no difficulties arise in the calculation of the elements of $\boldsymbol{\beta}_{R}$. In the case of $X_{3} C Y$-molecules (see Fig. 1d) with $X=\mathrm{H}$ or $\mathrm{D}$ and $Y$ a halogen atom, a multiplication factor of 1000 for the $C^{\prime}$ and $Y$ atom is very satisfactory, due to the low $X$-masses. It can be seen from Table $\mathrm{I}$ that the use of this reference molecule leads to results which agree within $1 \%$ with those obtained using a reference molecule with zero $X$-masses.

If the $X$-atom has a large mass e.g., $\mathrm{Br}_{3} \mathrm{CH}$, the multiplication factor must be 10000 or cven more (see Fig. 2 and Table II).

${ }^{1}$ If three or more atoms are situated on the main symmetry axis, only two adjacent nonterminal atoms must be replaced by heavy isotopes (e.g., the two $\mathrm{C}$ atoms in methylcyanide). 


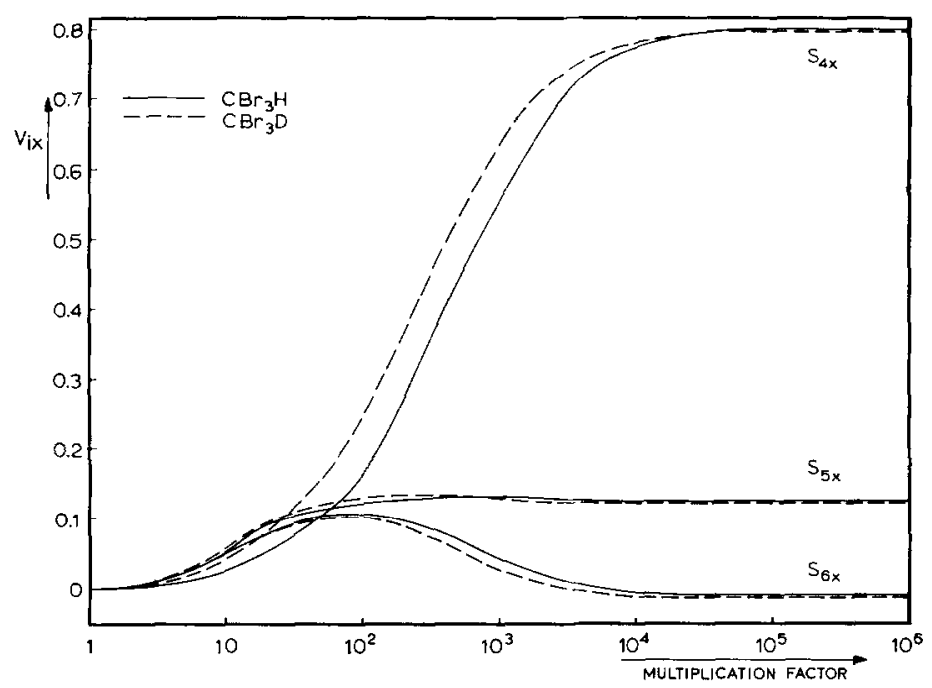

Fig. 2. Rotational correction terms $V_{i x}$ for $\mathrm{Br}_{3} \mathrm{CH} / \mathrm{D}$ as a function of the fartor by which the atoms on the main symmetry axis (C and H/D) were multiplicated. $S_{4 x}, S_{5 x}$, and $S_{6 x}$ are symmetry coordinates as defined in Table II.

\section{REMARKS ON THE CALCULATION OF ROTATIONAL CONTRIBUTIONS}

In order to ensure that the rotational correction terms can be interpreted unambiguously, it will be necessary that clear definitions are used with respect to:

1. The dipole-moment direction. The dipole-moment vector is directed from the center of the negative charges to the center of the positive charges. $\left(\partial \mu_{\xi} / \partial S\right)$ and $V_{\xi}$-values are positive when positive charge is displaced in the positive $\xi$-direction $(\xi=x, y$, or $z)$.

2. The Cartesian coordinate axes. The Cartesian coordinate axes are defined so as to form a right-hand coordinate system (see Fig. 1 ) $\mathbf{i} \times \mathbf{j}=\mathbf{k}$, and so on, cyclically.

3. The direction of rotation. A rotation about the $\xi$-axis is defined as positive when its rotation-vector $\omega_{\xi}$ points along the positive $\xi$-axis. As a consequence the general form of the $\beta$-matrix elements can be given as follows.

$$
\begin{array}{lll}
\rho_{1}\left(T_{x}\right): \beta_{1,3 i-2}=m_{i} / m_{\mathrm{tot}}, & \beta_{1,3 i-1}=0, & \beta_{1,3 i}=0, \\
\rho_{2}\left(T_{y}\right): \beta_{2,3 i-2}=0, & \beta_{2,3 i-1}=m_{i} / m_{\mathrm{tot}}, & \beta_{2,3 i}=0, \\
\rho_{3}\left(T_{z}\right): \beta_{3,3 i-2}=0, & \beta_{3,3 i-1}=0, & \beta_{3,3 i}=m_{i} / m_{\mathrm{tot}}, \\
\rho_{4}\left(R_{x}\right): \beta_{4,3 i-2}=0, & \beta_{4,3 i-1}=-m_{i} z_{0 i} / I_{x x}, & \beta_{4,3 i}=m_{i} y_{0 i} / I_{x x} \\
\rho_{5}\left(R_{y}\right): \beta_{5,3 i-2}=m_{i} z_{0 i} / I_{y y}, & \beta_{5,3 i-1}=0, & \beta_{5,3 i}=-m_{i} x_{0 i} / I_{y y}, \\
\rho_{6}\left(R_{z}\right): \beta_{6,3 i-2}=-m_{i} y_{0 i} / I_{z z}, & \beta_{6,3 i-1}=m_{2} x_{0 i} / I_{z z}, & \beta_{6,3 i}=0,
\end{array}
$$

where $i=1,2, \ldots, N$

$\xi_{0 i}=$ the equilibrium $\xi$ distance of atom $i$ to the center of mass $(\xi=x, y, z)$.

$I_{x x}, I_{y y}, I_{z z}$ are the principal moments of inertia. 
4. The symmetry coordinates. The symmetry coordinates are built up from internal coordinates (e.g., $\Delta r_{i}, \Delta \alpha_{i}$ ). The internal coordinates are taken as increments in bondlength and interbondangles. If scaled symmetry coordinates are used, the scaling factors must be clearly indicated. The scaling factors influence some of the $U$-matrix elements and consequently the rotational correction terms (see Eq. (9)).

Table V. Pyramidal $\mathrm{X}_{3} \mathrm{Y}$ molecules.

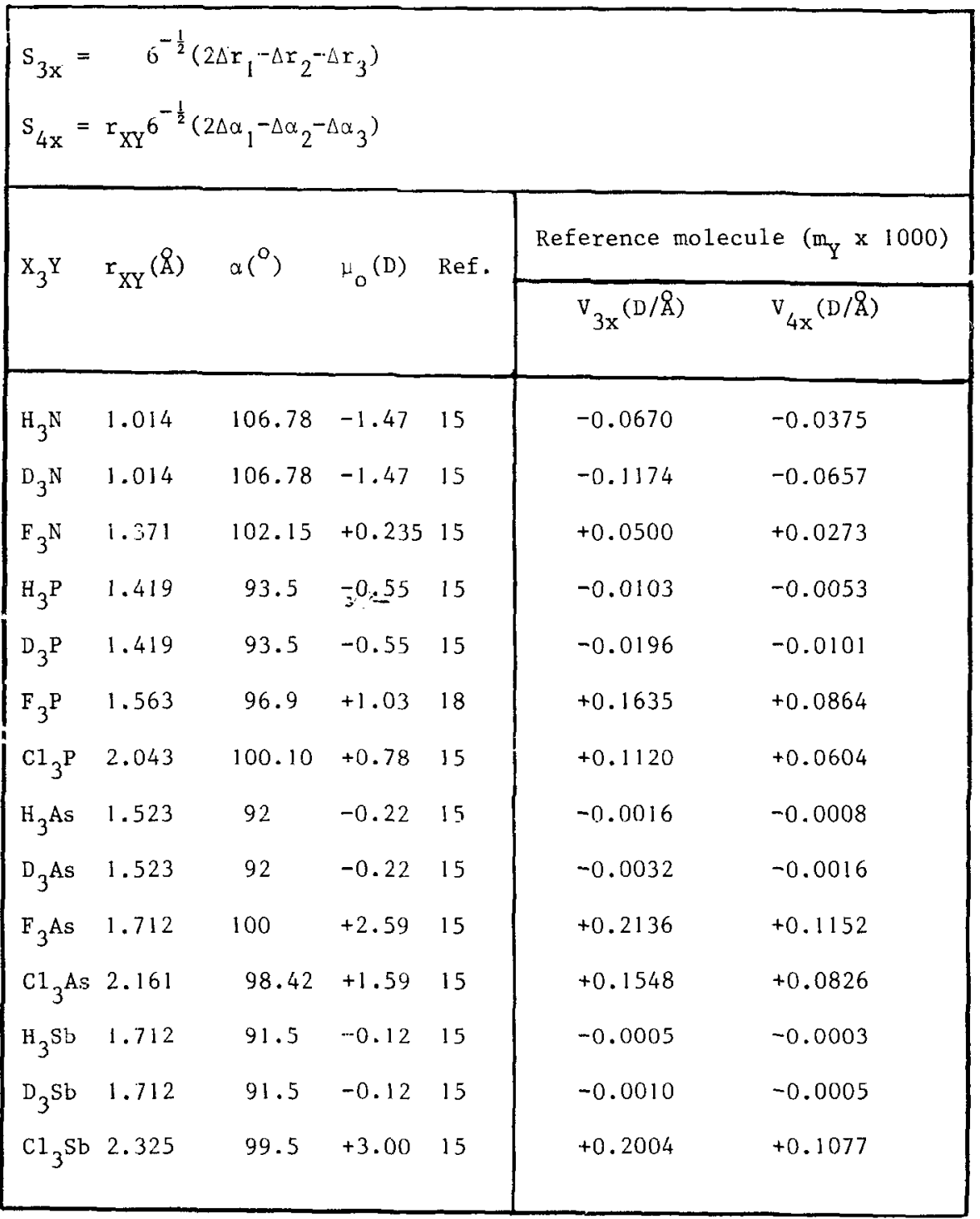




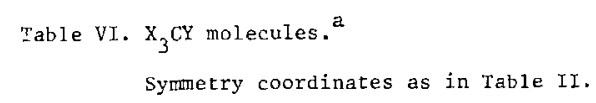

\begin{tabular}{|c|c|c|c|c|c|c|c|}
\hline \multirow[t]{2}{*}{$\mathrm{x}_{3} \mathrm{CY}$} & \multirow[t]{2}{*}{${ }^{r}{ }_{C X}(\AA)$} & \multirow[t]{2}{*}{$\mathrm{r}_{\mathrm{CY}}(\AA)$} & \multirow[t]{2}{*}{$a\left({ }^{\circ}\right)$} & \multirow[t]{2}{*}{$\mu_{0}(\mathrm{D})$} & \multicolumn{3}{|c|}{$\begin{array}{l}\text { Rotational corrections } \\
\text { Reference molecule }\left(\mathrm{m}_{\mathrm{C}}, \mathrm{m}_{\mathrm{Y}} \times 100000\right)\end{array}$} \\
\hline & & & & & $v_{4 x}$ & $v_{5 x}$ & $v_{6 x}$ \\
\hline $\mathrm{F}_{3} \mathrm{CH}$ & 1. 332 & 1.098 & 108.80 & +1.65 & +1.3021 & +0.2640 & -0.0710 \\
\hline $\mathrm{F}_{3} \mathrm{CD}$ & 1.332 & 1.098 & 108.80 & +1.65 & +1.2629 & +0.2385 & -0.0820 \\
\hline $\mathrm{CI}_{3} \mathrm{CH}$ & 1.767 & 1.073 & 108.40 & +1.01 & +0.8140 & +0.1473 & -0.0250 \\
\hline $\mathrm{Ci}_{3} \mathrm{CD}$ & 1.767 & 1.073 & 108.40 & +1.01 & +0.8061 & +0.1415 & -0.0292 \\
\hline
\end{tabular}

\section{CALCULATIONS AND CONCLUDING REMARKS}

Using the reference molecule as proposed here, rotational contributions were calculated for the following types of molecules. Bent $X_{2} Y$ molecules: $C_{2 v}$ (see Table III and Fig. 1a); $X_{2} C Y$ molecules: $C_{2 v}$ (see Table IV and Fig. 1b); pyramidal $X_{3} Y$ molecules: $C_{3 v}$ (see Table V and Fig. 1c); and $X_{3} C Y$ molecules: $C_{3 v}$ (see Table VI and Fig. $1 \mathrm{~d}$ ).

It is evident from Table $I$ that the results obtained with our reference molecule are the same as those obtained with Crawford's reference molecule. The reference molecule as proposed here leads to correct rotational contributions for all small molecules, with the exception perhaps of triatomic linear ones and molecules in which no $n$-fold axis $(n \geqslant 2)$ is present. In the case of those molecules difficulties arise, by applying a Crawford's type reference molecule as well as our type of reference molecule. The choice of a rotational free molecule is not quite clear in such cases, which leads to some arbitrariness in the absolute rotational contribution.

However, if the molecular symmetry is $C_{z}$ or lower a sensible choice can sometimes be made (e.g., the $\mathrm{C}$ and $\mathrm{O}$ atoms in methanol, the $\mathrm{C}-\mathrm{O}$ bond being almost a 3 -fold axis). In "large" molecules (eight or more atoms) the rotational contributions can be ignored.

\section{ACKNOWLEDGMENT}

Our thanks are due to Dr. B. L. Crawford, Jr., for reading the manuscript prior to publication.

REceived: October 30, 1974

\section{REFERENCES}

1. B. Crawford, JR., J. Chem. Phys. 20, 977 (1952).

2. A. D. Dickson, I. M. Mills, and B. Crawford, JR., J. Chem. Phys. 27, 445 (1957).

3. B. Crawford, Jr., J. Chem. Phys. 29, 1042 (1958). 
4. E. B. Wilson, JR., J. C. Decius, and P. C. Cross, "Molecular Vibrations," McGraw-Hill, New York, 1955.

5. J. Overend, "Infrared Spectroscopy and Molecular Structure" (M. Davies, Ed.), Chap. X, Elsevier, New York, 1963.

6. J. W. Russell, C. D. Needham, and J. Overend, J. Chem. Phys. 45, 3383 (1966).

7. M. J. Hopper, J. W. Russell, ANd J. Overend, J. Chem. Phys. 48, 3765 (1968).

8. D. C. McKean, R. E. Bruns, W. B. Person, and G. A. Segai, J. Chem. Phys. 55, 2890 (1971).

9. R. E. Bruns and R. K. Natr, J. Chem. Phys. 58, 1849 (1973).

10. M. J. Hopper, J. W. RusSeld, ANd J. Overend, Spectrochim. Acta 28A, 1215 (1972).

11. R. E. Bruns and W. B. Person, J. Chem. Phys. 58, 2585 (1973).

12. J. Aldous and I. M. Mulis, Spectrochim. Acta 19, 1567 (1963).

13. S. Kojima, K. Tsukada, S. Hagiwara, M. Mitushima, and T. Ito, J. Chem. Phys. 20, 804 (1952).

14. "Interatomic Distances" (L. E. Sutton, Ed.), Special Publication no. 11, The Chemical Society, London, 1958.

15. W. Gordy, W. V. Smith, and R. F. Trambarulo, "Microwave Spectroscopy," Wiley, New York, 1953.

16. K. Takagi and T. Oka, J. Phys. Soc. Japan 18, 1174 (1963).

17. V. W. Laurie, D. T. Pence, and R. H. Jackson, J. Chem. Phys. 37, 2995 (1962).

18. E. Hirota aNd Y. Morino, J. Mol. Spectrosc. 33, 460 (1970).

19. S. N. Ghosh, R. Trambarulo, and W. Gordy. J. Chem. Phys., 20, 605 (1952). 